\title{
A SURVEY ON "ENERGY EFFICIENT TECHNIQUES OF 5G MIMO: AN INNOVATIVE FUTURE OF WIRELESS COMMUNICATION"
}

\author{
Aaishwarya Soni' ${ }^{1}$, Mrs. Megha Soni ${ }^{2}$ \\ Student of M.Tech (Digital Communication), Electronics and communication engineering, Babulal Tarabai Institute of \\ Research and Technology, Sagar (M.P.), India ${ }^{1}$ \\ HOD and Guide, Department of Electronics and communication engineering, Babulal Tarabai Institute of Research and \\ Technology, Sagar (M.P.), India ${ }^{2}$
}

\begin{abstract}
The enormously enhancing the evolution of forthcoming generations, the fifth-generation wireless technology continuously developed and extent to more progressive. The field of mobile technology provides improved network capacity, low latency, great availability, more reliability with intense speed. This paper introduces an overview of fifth-generation wireless technology accompanied by five other major technologies of $5 \mathrm{G}$ namely millimeter wave, small cells, Massive MIMO, Beamforming, and Full-duplex respectively. In addition, Energy Efficiency (EE) is an enormous strategy that immensely declines the circuit power consumption in fifth-generation cellular networks. This consequence is enhanced due to the rising number of antennas in massive multiple-input multiple-output (MIMO) systems. Energy efficiency has now become a fundamental aspect in the framework of communication networks. With the arrival of the fifth generation of wireless networks, with millions of additional base stations and billions of connected devices, the demand for energy-efficient system design and operation will be even more fascinating. Further, this paper outlines the various research work related to energy efficiency including the papers published in this special issue, and considers the most compelling research challenges to be discussed in the upcoming generation.
\end{abstract}

Keywords: 5G,Massive MIMO, Millimeter wave, small cells, Beamforming, Full duplex, heterogeneous networks, Energy Efficiency (EE).

\section{I.INTRODUCTION}

The area of wireless communication enhanced technically in advancing from 1st Generation to 5th Generation as $1 \mathrm{G}$ to 5G. These technologies comprise GSM, WCDMA, LTE, Wi-MAX, MIMO, and mm Waves facilitate voice calls, online data delivery with high speed, and texting. Now, in forthcoming generations, massive communication becomes possible between far things. It also employs voice, text, and data at very high speed. The comprehensive vision of the Future Generation Communication Systems (FGCS) is to accomplish high bandwidth, energy efficiency, low latency, and additional data delivery to allow good broadband services. 5G can hold up to a million devices per square kilometer, while $4 \mathrm{G}$ hold only up to 100,000 devices per square kilometer.

More than speed, the lead benefit of $5 \mathrm{G}$ is its low latency. Latency refers to the timeline between the device asking the network and getting responses. 5G approximately eradicates the timeline. It means that the surgeon could be 1,000 miles away from the patient can control the equipment. It will favor the Internet of Things (IoT). 5G will also allow you to transmit your tactile or touch sensation in real-time. $5 \mathrm{G}$ empowers connecting vehicles and co-operating drones. It will also enable virtual reality, best streaming, guaranteed connectivity, and extraordinary quality. With 5G, the number of devices connected to the network will increase tremendously. 5G is essentially Mobile Telecom Infrastructure, which is design to associate everyone in everything together comprising machines, devices, and objects. 5G carries three crucial things such as huge channels to speed up data, lower latency to be more compassionate, and the ability to connect many devices at a time.

Compare to 4G, 5G has significantly lower latency ( $<10$ milliseconds) and at least 10 times faster than 4G. 5G has more capacity that 4G such that it can handle 1000 times more traffic than $4 \mathrm{G}$. It also used better spectrum efficiency. Electronic devices including cellular phones and wireless routers use definite frequencies on the electromagnetic spectrum. The frequencies used by $4 \mathrm{G}$ telecom operators are typically up to $3 \mathrm{GHz}$. As more and more devices are getting online, the current $4 \mathrm{G}$ frequency band is becoming crowded. Overcrowding within a small band of frequencies leads to dropped connections and slower speed. A major solution to overcome overcrowding is to allow devices to 


\section{International Journal of Advanced Research in Computer and Communication Engineering}

Vol. 10, Issue 5, May 2021

\section{DOI 10.17148/IJARCCE.2021.105148}

operate the frequencies in other bandwidth i.e., open up additional frequencies for telecommunication. Fifth Generation cellular network can exactly perform this. It operates under more frequency bands from $3 \mathrm{GHz}$ to $39 \mathrm{GHz}$ and possibly up to $300 \mathrm{GHz}$ in the future. The use of this portion of the spectrum means more frequencies would be available for many more devices to communicate. In addition to getting more frequencies, 5G maintain its own set of trend new technologies as well. Evolution of generations of the communication standards demonstrated in figure 1.

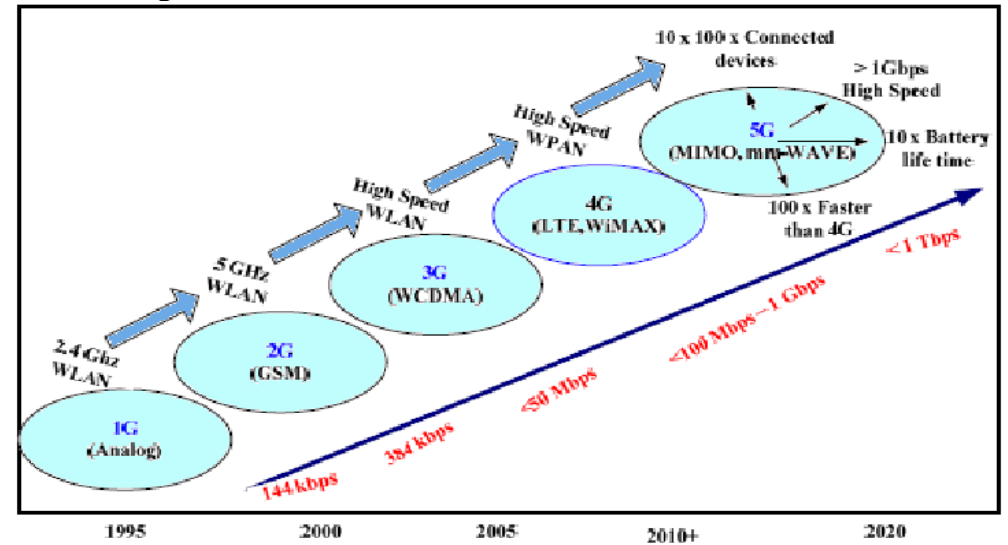

Fig.1 Evolution of generations of the communication standards.

\section{A. $1 \mathrm{G}$}

$1 \mathrm{G}$ stands for first generation network includes the use of analog radio signals at the data rate of 9.6kbps to offer analog voice services i.e., Analog Telephony. In $1 \mathrm{G}$, the analog signals are used with no data capabilities. It is commonly called an analog transmission technology that provides basic voice service. AMPS provides the first commercial cellular phone concept. The analog radio signals are employed with no data capacities, though digital signaling is managed to connect the radio towers to the rest of the telephone system by modulating (frequency modulation (FM)) the voice calls to a more immense frequency of about $150 \mathrm{MHz}$.

Alternatively, frequency division multiplexing (FDM) is used to isolate the bandwidth into definitive frequencies that are designated to respective calls. The cell size for a conventional $1 \mathrm{G}$ network is about $2-20 \mathrm{~km}$.

The $1 \mathrm{G}$ network is set up on analog signals/protocol technology (FM) that determines one prevailing issue such as susceptibility to interference, which diminishes call quality. In addition, there was a lack of security due to no advanced encryption methods includes in analog signals. The $1 \mathrm{G}$ is entangled by limited capacity, huge phone size, lack of voice quality, less battery life, and frequent call dropping. It provides up to $30 \mathrm{KHz}$ spectrum flexibility.

\section{B. $2 \mathrm{G}$}

$2 \mathrm{G}$ stands for second generation network developed on GSM standards. This generation upgrades as, radio channel used by $1 \mathrm{G}$ network is analog, while $2 \mathrm{G}$ network id digital. $2 \mathrm{G}$ was able to provide a significantly improved voice quality. It is the first digital data service in the advancement of cellular network technology. It provides secure short message service (SMS) and multimedia messaging service (MMS) services and becomes digitally encrypted to overcome some of the limitations of $1 \mathrm{G}$. $2 \mathrm{G}$ capabilities are achieved by allowing multiple users on a single channel via, multiplexing. To enhance connectivity, $2 \mathrm{G}$ was able to provide a semi-global roaming system. In advance, the $2 \mathrm{G}$ global system for mobile communications (GSM) specification supports cell sizes of up to $35 \mathrm{~km}$ using macro, micro, pico, or femtocells.

\section{C. $3 \mathrm{G}$}

3G stands for third generation network support broadband and multimedia services. It utilizes a new technology called UMTS. Urged slightly by the growth in internet and IP network technology, 3G architecture strengthens support for an embellished data rate (throughput speed) and QoS. 3G technology involves the services such as global roaming and enhanced voice quality. It enables mobile telephony. Due to the consumption of more power as compared to previous generations, it is less economical to set up and operate. Thus, it is a major drawback in 3G Technology. Furthermore, 3G UMTS is backward adaptable with prior generations of cellular wireless technologies through its capacity to endure in distinction with the estate GSM or AMPS technology. The progression from UMTS through High-Speed Packet Access (HSPA) and expanded HSPA (HSPA+) significantly brought enlarged end-to-end network performance and ultimately brought to the development of the next generation of networks i.e. $4 \mathrm{G}$.

D. $4 \mathrm{G}$

$4 \mathrm{G}$ stands for fourth generation network that represents the generation of mobile cellular communication technology provide the broadband data transmission and broadcasting, in addition to very high-volume voice users. The architecture of 4G is based on , 4G Long Term Evolution (LTE) network, which is designed with to provide support for 


\section{International Journal of Advanced Research in Computer and Communication Engineering}

Vol. 10, Issue 5, May 2021

\section{DOI 10.17148/IJARCCE.2021.105148}

packet-switched traffic with seamless mobility, QoS, and minimal latency. This approach allows for the support of all services (data, voice, multimedia) through packet connections. The potential to accomplish the least latency, upgrading in Multiple Input Multiple Output (MIMO) techniques through discrete Radio Access Technologies (RATs) such as Orthogonal Frequency Division Multiple Access (OFDMA) and Single Carrier Frequency Division Multiple Access (SCFDMA) are elements of the crucial concerns and accomplishments of 4G technology. Diverse contemporary approaches such as carrier aggregation, relaying, and Coordinated MultiPoint (CoMP) transmission and/or reception conclusively constructed and carried out to administer further enhanced peak data rates, support for heterogeneous network formation, and spectrum flexibility amongst other competencies are astonishing achievements contributed by $4 \mathrm{G}$ technology. The areas of multicasting and interference mitigation are other major enhancements brought by the $4 \mathrm{G}$ era. It involves multicasting services i.e. evolved Multimedia Broadcast Multicast Service (eMBMS). The 4G technology provides the fastest speed, reduced latency, and crystal-clear voice calls. The $4 \mathrm{G}$ offers speed up to $1.5 \mathrm{Gbps}$.

\section{E. 5G}

The next generation wireless technology is $5 \mathrm{G}$ i.e., Fifth Generation Technology. 5G has the capability to reinforce an extensive number of connections concurrently while advancing speed, latency, reliability, and power consumption for handsets and Internet of Things (IoT) devices. It provide a speed of 100 times faster than LTE, with 1-10 Gb/s with Massive connections of 100 - fold increase of supported devices to $1 \mathrm{mn}$ devices $/ \mathrm{km} 2$. A $5 \mathrm{G}$ Technology provide Latency from $20 \mathrm{~ms}$ to $1 \mathrm{~ms}$ Enhanced Mobile Broadband (EMBB), which provides faster data rates across wide coverage areas to improve various functions, including ultra-HD video streaming — Ultra-Reliable, Low-Latency Communication (URLLC), which broaden the speed and Quality of Service(QoS) in crucial functions, such as the control of robots and drones. Likewise, Massive Machine-type Communication (MMTC), associates the automatic generation, transmission, and processing of data among diverse machines without human invasion.

Second Generation cellular telecom networks based on GSM standards were commercially launched in 1991 in Finland. It could deliver a data rate of up to $9.6 \mathrm{Kbps}$. $2 \mathrm{G}$ introduced data services for mobile, starting with the SMS text messages. The three primary benefits of $2 \mathrm{G}$ networks over their predecessors were:

1. Phone conversations was digitally encrypted.

2. It was significantly more efficient on the spectrum.

3. It allowed far greater mobile phone penetration level.

Further, to achieve higher data rates GSM carriers started developing a service called General Packet Radio Service (GPRS). This system overlaid a packet switching network on the existing circuit switched network. GPRS could transmit data rates up to $160 \mathrm{Kbps}$. The phase after GPRS is called Enhanced Data Rats for GSM Evolution (EDGE). It introduced 8 PSK modulation and could deliver data rates up to 500Kbps using the same GPRS infrastructure. Now the internet is becoming more popular and data services becoming more prevalent. Development of 3G, includes 3GPP UMTS, the Universal Mobile Telecommunications system succeeded EDGE in 1999. This system uses Wideband CDMA (W-CDMA) to carry the radio transmission, and often the system is referred to as WCDMA.Further, the production of truly global standards, the collaboration of both GSM and UMTS was expanded from ETSI to encompass regional Standards Development Organizations. The fortunate establishment of such an extensive and complicated system stipulation expected a well-structured framework. This gives birth to 3GPP and worked under the observation of ITU-R. ITU-R is one of the sectors of ITU, which manage the international radio-frequency spectrum and to ensure the effective use of spectrum ITU-R defines technology families and associate specific parts of the spectrum with these families. ITU-R also proposed a requirement for radio technology. Evolution of 3GPP, started from GSM to Long Term Evolution Advanced. 3G wireless network aims to provide a minimum data rate of $2 \mathrm{Mbps}$ for stationary or walking users, and $384 \mathrm{Kbps}$ for moving vehicles. The Fourth generation system or Long Term Evolution (LTE) contain some significant features as follows:

- $\quad$ Reduced delays, for both connecting establishment and transmission latency.

- Increased user data throughput.

- Increased cell-edge bit rate, for uniformity of service provision.

- $\quad$ Reduced cost per bit, implying improved spectrum efficiency.

- $\quad$ Simplified network architecture

- $\quad$ Seamless mobility, including different radio-access technologies.

- $\quad$ Reasonable power consumption for the mobile terminal.

The three fundamental technologies that have shaped the LTE radio interface design were :

1. Multicarrier technology

2. Multiple- antenna technology

3. The applications of packet switching to the radio interface.

In advancement, from services to multi-services i.e., from LTE Advanced to next Generation communication system is 5th Generation. The features of the 5th Generation includes: 


\section{International Journal of Advanced Research in Computer and Communication Engineering}

Vol. 10, Issue 5, May 2021

\section{DOI 10.17148/IJARCCE.2021.105148}

- $\quad$ Pervasive networks, in which users can concurrently be connected to several wireless access technologies and seamlessly move between them.

- Group cooperative relay, is a technique, considered to make high data rates available over a wider area of the cell.

- Cognitive radio technology, is a technique that would enable the user equipment / handset to look at the radio landscape in which it is located and choose the optimum radio access network, modulation scheme, and other parameters to configure itself to gain the best connection and optimum performance.

- Smart antennas are another major element of any 5G cellular system. Through this, it is the potential to modify the beam direction to facilitate communications that are more direct and inhibit interference and advance overall cell capacity.

While it can be argued that $3 \mathrm{G}$ and $4 \mathrm{G}$ technologies can connect peoples and partly things (objects or artifacts) in the case of 4G long term evolution (LTE) / LTE-Advanced (LTE-A). The developmental efforts to date towards the realization of $5 \mathrm{G}$ make it look increasingly indisputable that $5 \mathrm{G}$ will be able to connect everything, providing a seamless, coalescing connectivity for at least the next decade and possibly beyond. In other words, it suffices to assert that the advent of $5 \mathrm{G}$ will provide limitless access anywhere, at any time, for anyone, and anything. This is partly because this anticipated generation of technology, if successful, would create a unified air interface in establishing endto-end connectivity between mundane things such as smartphones, fridges, freezers, boilers, cars, wearable, utility meters, and many more. To put it in technical terms, 5G brings a world of appreciably enhanced mobile data broadband, ultra responsiveness, ultra-reliability, ultra-low latency, ultra-fast data rate, and enormous MTC/M2M or IoT capabilities. In 5G, the essential ingredients of radio resource allocation (a key component of RRM) including latency, throughput, reliability, QoS, and QoE are expected to be significantly optimized to entirely new, unprecedented levels. Several other key concepts, techniques, and schemes are CoMP, SC-FDMA, OFDMA, frequency division duplex time division duplex carrier aggregation (FDD-TDD CA), etc. One other key aspect of 5G is the evolution from cell centricity into device centricity, which exploits and harnesses intelligence at the device side (human or machine) such as via device-to-device (D2D). The target of 5G beyond 2020 is to fully commercialize 5G infrastructure deployment. Some advantages of $5 \mathrm{G}$ are as follows:

- It provides intense speed.

- Low latency: Latency is the time between accessing an event and the response to it. Low latency is the envy, relatively spontaneous, feedback that makes all from gaming to downloading videos a c easy accomplishment. A huge file, for example, that would take over $25+$ hours to download with $3 \mathrm{G}$ technology grabs less than four seconds to download with $5 \mathrm{G}$.

- $\quad$ Peer-to-peer (P2P) communications: One can easily transfer data between devices in a matter of seconds.

- Vehicle-to-vehicle communications (V2V): 5G communication makes it possible for cars linked to smart traffic management systems to exchange information such as location, speed, and destination.

- It provides better gaming experiences.

- It provides enhanced cloud gaming technology.

- Improved virtual reality: With 5G advancements, response time is whittled down to a mere couple of milliseconds.

- $\quad$ It provides Robotic medical advancements.

- $\quad$ This technology provides immense development in the field of Robotic surgeries.

- It provides an evolution of IoT.

\section{II.TECHNOLOGIES OF 5 G}

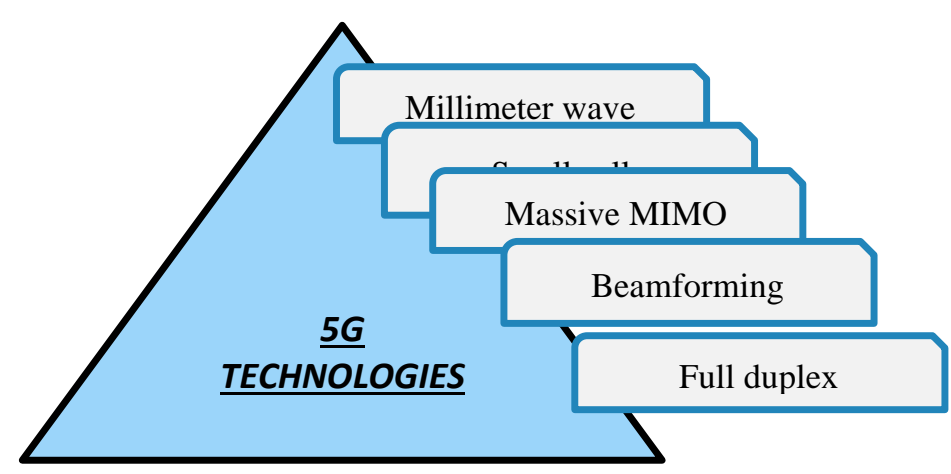

Fig.2 Major technologies of 5G. 


\section{International Journal of Advanced Research in Computer and Communication Engineering}

Vol. 10, Issue 5, May 2021

DOI 10.17148/IJARCCE.2021.105148

The five major technologies in 5G are Millimeter wave, small cells, Massive MIMO, Beamforming, Full duplex illustrated in figure 2. The electromagnetic waves are used while communication, which is classified as seven types, namely: Radiowaves for instant communications, Microwaves for data and heat, Infrared rays for invisible heat, Visible rays allow seeing, Ultraviolet rays are energetic light, X-Rays can penetrate our body and Gamma rays are nuclear energy.

The communication between the two stations in mobile communication refers to Air Interface or Access Mode. For wide-area coverage, 5G networks operate up to three frequency bands: Low Band 5G, Mid band 5G, and High Band $5 \mathrm{G}$. frequency band of $5 \mathrm{G}$ network shown in table 1 .

TABLE I FREQUENCY BANDS OF 5G NETWORKS

\begin{tabular}{|c|c|c|}
\hline Frequency bands & Range & Features \\
\hline Low Band 5G & $600 \mathrm{MHz}-850 \mathrm{MHz}$ & $\begin{array}{l}\text { - } \\
\text { Similar to } 4 \mathrm{G} \\
\text { The low band cell towers have a coverage } \\
\text { area range similar to } 4 \mathrm{G} \text { cellphone towers. } \\
\text { - } \quad \text { Speeds are } 30-250 \mathrm{Mbps}\end{array}$ \\
\hline Mid Band 5G & $2.5 \mathrm{GHz}-3.7 \mathrm{GHz}$ & $\begin{array}{l}\text { - } \\
\text { range. } \\
\text { Frequencies are used in the Microwave } \\
\text { kilometers in radius. } \\
\text { - } \quad \text { Speeds are between } 100-900 \mathrm{Mbps}\end{array}$ \\
\hline High Band 5G & $25 \mathrm{GHz}-39 \mathrm{GHz}$ & $\begin{array}{ll} & \text { Frequencies are used in the Microwave } \\
\text { range. } & \\
\text { - } & \text { Limited coverage range and needing small } \\
\text { cells. } & \end{array}$ \\
\hline
\end{tabular}

\section{A. Millimeter waves}

Millimeter waves broadcast at frequencies between 30 gigahertz to 300 gigahertz. As, they vary in their wavelengths from 1 millimeter to 10 millimeters hence, named Millimeter waves. In comparisons with radio waves, used in today's smartphones measure 1,000 times longer than Millimeter waves. So far, Millimeter waves were used in satellite communication as well as in RADAR operations.

Some cellular providers become bigger to use them to exchange data between two stationary points. However, using Millimeter waves to connect mobile users with nearby base stations is entirely a new approach. The major problem is that it cannot easily travel through the buildings and obstacles. In addition, trees, plants, and rain can easily absorb Millimeter waves. This consequence can be overcome by another 5G technology such as Small Cells.

\section{B. Small cells}

Small cells are mini portable base stations, which require very low power. Millimeter waves cannot penetrate plants and buildings. Thus to prevent signals from the drop telecom operators may install thousands of small cell base stations in a town to create a dense network. Each base station will receive signals from other base stations and may forward data to users. A dense network of small cells increases both ratios per subscribers and provides improved signal quality for more efficient data transfer. A shorter distance between radio sizes also helps to overcome the short signal distance reach of millimeter waves or the other higher frequencies in the $5 \mathrm{G}$ radio spectrum.

Due to the combinational intricacy of low-complexity joint antenna selection and user, a small cell becomes the leading solution to increasing the data capacity of the network. Small cells are small to be enough to be installed on the light poles or the top of the residential buildings. This dense network provides more targeting and efficient user spectrum. Spectrum refers to the number of frequencies available to communicate. This is a very scares and expensive resource. Installing more base stations in 5G small cells means the frequency that one base station uses with the mobile devices in one area can be reused by another base station in a different area to serve another user. It shows that a smaller cell leads to greater use of frequencies and hence provides high spectral efficiency.

In early times, we had radiotelephones in cars. In this system, there was one central antenna tower in a city and perhaps 25 channels available on that tower. Since the center antenna is so far, the phone in the car needed a powerful transmitter to reach the antenna far away. In addition, limited the number of available channels met that many peoples can use it simultaneously. Meanwhile, cell phones came out, which divide the city into many small cells, which allow the frequency reused across the city such that many people can use cell phones simultaneously. In the typical analog cell phone system in the US, a carrier was granted about 800 frequencies in a city. Carriers then divided the city into many cells and reused frequencies across them to allow many users to use cell phones simultaneously. In small cells, it 


\section{International Journal of Advanced Research in Computer and Communication Engineering}

Vol. 10, Issue 5, May 2021

DOI 10.17148/IJARCCE.2021.105148

is difficult to set up a large number of small cells required to build up 5G network in rural areas. In addition, for broadcasting over millimeter waves $5 \mathrm{G}$ base stations will also help to have many more antennas fitted into the base stations of today's cellular networks. Fitting many antennas into a smaller sizes is a big issue, which is overcome by another 5G technology called Massive MIMO.

\section{Massive MIMO}

Since length of an antenna is half of the wavelength of the waves it emits. Also, the wavelength is inversely proportional of frequency, so, higher the frequency in $5 \mathrm{G}$, the smaller will be the size of the antenna. If the size of the antenna is small we can fit many more antennas into one array, which means we can build small cell base stations with a large number of antennas fitted into them relatively more easily. 4G base stations have about a dozen antenna ports. These ports manage all the cellular traffic, out of the 12/8 ports are for transmitters and 4 ports are for receivers whereas $5 \mathrm{G}$ base stations can support about 100 ports, which means many more antennas can fit into a single array. This increased capability means that the base station can send and receive signals from many more users simultaneously. Thus, the capacity of mobile networks increases. Continuously, using two or more antennas for input and output signals that are receiving and sending signals is called MIMO, which stands for Multiple Input Multiple Output.

In massive MIMO, dozens of antennas are used in a single array. Hence, the word massive was used along with the concept of MIMO. The most commonly seen definition of massive MIMO is that it is a system where the number of antennas exceed the number of users. In practice, it is called massive when there are 64 or more logical antenna ports in an array in a base station.

\section{Full duplex}

In addition, to using massive MIMO, $5 \mathrm{G}$ engineers also trying to increase throughput and decrease latency by using the technology called full duplex, which modifies the way the antenna transmits and receives the signal and data.

In $3 \mathrm{G}$ and $4 \mathrm{G}$ networks, the two basic configurations for sending and receiving signals such as Frequency Division Duplexing (FDD) and Time Division Duplexing (TDD) are used. In FDD, transmission and reception happen at two different frequencies. The second configuration refers to Time Division Duplexing (TDD). In TDD, the send signal is separated from receive signal by using different time slots in the same frequency band i.e., the send and receive signals take turns, sends or uplink signals connects for few seconds and, the downlink or receive signals connects for the next few seconds. The full duplex has the ability of the transceiver to transmit and receive data at the same time for the same frequency. These technologies can potentially double the network capacity in a full duplex. The spectrum efficiency improved greatly in comparison with half-duplex (HD). But, there is a problem in the full duplex that is signal cell interference $\mathrm{Si}$ in the signal leakage from transmitter to its own receiver there are some techniques to overcome this problem which are as follows:

1. Propagation Domain SI suppression,

2. analog domain SI cancellation,

3. Digital domain SI cancellation

However, there are some other obstacles in achieving theoretical full duplex such as Residual cell interference, Traffic constraints, and Intracellular and Intercellular interference.

\section{E. Beamforming}

MIMO has a big limitation. The Antenna sends the signals in every direction at once. Therefore, when so many antennas are positioned so close to each other, it causes interference in signals emitting by different antennas. To address this challenge, there is another technology called Beamforming. Instead of broadcasting in every direction, a 5G base station sets a focused beam of data to a particular user refers to as Beamforming. Many times, the terms Massive MIMO and beamforming are used interchangeably. Further, in reality, Beamforming is used in massive mimo. Beamforming is a subset of massive MIMO. Beamforming has its subcategories, which are as follows:

1. Digital Beamforming is also known as Precoding.

2. Analog Beamforming.

3. Hybrid Beamforming.

Massive MIMO used many antennas. Since these antennas are located too closely to each other; there is interference among the signals sent out by these antennas. To avoid interference, Beamforming is used. Beamforming controls the direction of the wavefront and can be achieved by appropriate magnitude and phase of the signal transmitted by an individual antenna in an array. In other words, the same signal is sent from different antennas but there is a phase difference or space between them of at least half wavelength. This causes constructive interference of signal waves in one direction and causes destructive interference in all other direction i.e., a beam or a lobe is formed in one direction while rest in another direction. At the receiving end, the user gets multiple copies of the same signal, one each from 


\section{International Journal of Advanced Research in Computer and Communication Engineering}

Vol. 10, Issue 5, May 2021

DOI 10.17148/IJARCCE.2021.105148

different antennas. However, depending upon the receiver via tower these multiple signals may be in opposite phases, and get destructive interference and hence cancel out each other or get constructively interference to create a stronger signal.

Since, antenna contains a radiating element, which emits electromagnetic radiation in all directions. However, instead, focus the radiation in all directions, if the user needs to focus the radiation in one direction then, there is a need of more than one antenna radiating element to feed them with the same signal. It refers to the single antenna with a more directed signal. For example, if we use two radiating elements placed next to each other, we get twice the energy in one direction and less energy going sideways in other directions. This two times energy is known as three decibels. Similarly, if we have four radiating elements and feed them with the same signal, we still have one antenna but we will get four times the energy going forward in one direction known as six decibels. The problem with this is that the receiver has to be in front of the antenna, if the receiver wants to be on the side the signal will not reach it.

Therefore, to target the beam in the same direction, we need to change the direction of the antenna or we need to feed each radiating element with the same signal but with different phases. If the signal to all radiating elements is the same except that they have different phases, we will have constructive interference in one direction and there will be destructive interference in all other directions. Instead of different phases, we can also use different amplitudes to have constructive interference in a given direction and create different types of beams.

By varying, the input signals to the radiating elements one can create multiple beams as well. These beams could go in different directions. In fact, one can also create a beam, which goes directly a user standing at the foot of the base station. This process of sending multiple beams is known as Spatial Multiplexing or Spatial Division Multiple Access (SDMA) in which number of antennas is same as a number of input signals. One can multiplex as many beams as the number of antennas.

\section{III.RELATED WORKS}

\section{A. LITERATURE REVIEW}

The use of heterogeneous networks a novel approach for a substantial extension of the network capacity, with enough cost to provide a rising user experience, where small base stations (BSs) are covered within the main network depend on coverage demand. Energy Efficiency is an important parameter that provides an important design criterion, as it allows operations to remain practically affordable through regulated energy consumption levels. The orthogonal pilot sequences for all UEs in the same cell prevent channel estimation from affected by the interference of other UEs. In addition, to attain an applicable performance system with an enormous data rate were analyzed, such that it alters the data rate to reduce inter-cell interference and provide corresponding channel estimation [1].

Moreover, ascertaining the status of the CSI requires a correlation of the received pilot from every UE with the recognized pilot signal accompanied with that UE. The crucial requirement for maximizing EE is to compute the joint transmission power and optimal antenna selection under the affected pilot sequences. The number of selected antennas is imperative because when the RF chains are associated with the preferred antennas, those antennas deplete more power and negatively influenced EE. Also, the extended NoAs when transmitting pilot sequences to every UE evaluates the M channel in the DL. Further, the energy-efficient massive MIMO design issue is examined in both single-cell and multi-cell scenarios to select suitable parameters to enhance the overall system energy utilization [2].

Furthermore, many investigators inspected the number of RF chains, taking into account the consequence of channel estimation for large PRS when the NoAs increased. The majority of analysts focused only on decreasing RFs at the enlarged numbers of antennas to maximize EE.

Nevertheless, the study reported [3] research on maximum EE by considering the DL massive MIMO system with enhancing the NoAs, several UEs, and CPC for transmitting power by taking into consideration for channel estimation and PC. When the transmitter furnished with a consequential NoA, the computational capacity with a minimal number of RF chains and the joint antenna selection enhanced EE. Likewise, the low-complexity iterative solution in multi-cell Non-Orthogonal Multiple Access-based (NOMA) [4] proposed in 5G cellular networks to examine the decency of SINR-based manipulated transmit power allocation for distinctive UE.

Also, researchers surveyed the extreme EE [5] to carry out data rates with inadequate transmitted power by deciding the user's maximal transmit power and low rate constraints. The maximal EE attained the minimal required power and rate constraints relying on investigating the circuit power when the transmitter furnished with a critical NoA.

Many investigators examined the advancement of definite models and dynamic algorithms. By administering lower computational intricacy, optimal performance EE can be carried about by engaged identical transmit power allotted to every UE. Further, the study [6] suggests boosting cell capacity by initiating an abundant number of BSs by lowering the cost with a definite number of RF chains.

The combinatorial intricacy of low-complexity joint antenna selection and user scheduling can support high cell efficiency by alleviating inter-cell interference. The user selection and channel estimation enhanced based on mitigating 
the inter-user interference by exploiting a low-complexity iterative solution to attain optimal transmit power control and user selection. The summary of various previous related data, its features, and limitations is shown in table 2.

TABLE II SUMMARY OF PREVIOUS RELATED DATA, ITS FEATURES AND LIMITATIONS

\begin{tabular}{|c|c|c|c|}
\hline Year & Problem related to & Features & Limitations \\
\hline $\begin{array}{l}2011 \\
{[7]}\end{array}$ & $\begin{array}{l}\text { Problem of pilot } \\
\text { contamination in } \\
\text { Multi-cell systems. }\end{array}$ & $\begin{array}{l}\text { Used training pilot sequences. } \\
\text { - Achievable data rate by developing } \\
\text { a multi-cell MMSE. }\end{array}$ & - $\quad$ ZF and MMSE. \\
\hline $\begin{array}{l}2013 \\
{[8]}\end{array}$ & $\begin{array}{l}\text { Pilot contamination } \\
\text { effect, the equivalent } \\
\text { System model. }\end{array}$ & $\begin{array}{l}\text { - Used equivalent channel with pilot. } \\
\text { - Derived the rate in the lower } \\
\text { bound. }\end{array}$ & - Infinite NoA. \\
\hline $\begin{array}{l}2013 \\
{[9]}\end{array}$ & $\begin{array}{l}\text { More circuit power } \\
\text { dissipated by analogue } \\
\text { devices and a power } \\
\text { amplifier. }\end{array}$ & $\begin{array}{l}\text { Proposed a practical power } \\
\text { consumption model to maximise } \\
\text { EE. }\end{array}$ & $\begin{array}{ll}\text { - } & \text { Large NoA. } \\
\text { - } & \text { Proposed CPC }\end{array}$ \\
\hline $\begin{array}{l}2014 \\
{[10]}\end{array}$ & $\begin{array}{l}\text { EE aspects and the } \\
\text { associated gains of } \\
\text { implementing RAN-as-a- } \\
\text { Service }\end{array}$ & $\begin{array}{l}\text { low complexity decoding } \\
\text { framework } \\
\text { analysis of sparse code multiple } \\
\text { access (SCMA) scheme. } \\
\text { - evolution of the LTE-A network } \\
\text { toward 5G infrastructure }\end{array}$ & $\begin{array}{l}\text { Seamless coverage and } \\
\text { successful delivery of the } \\
\text { control signaling to all UE } \\
\text { in C-RAN is limited } \\
\text { - Lagrange Dual } \\
\text { Decomposition method is } \\
\text { proposed. }\end{array}$ \\
\hline $\begin{array}{l}2015 \\
{[11]}\end{array}$ & $\begin{array}{l}\text { - Pilot assignment } \\
\text { problem. } \\
\text { - Number of activated } \\
\text { antennas and power } \\
\text { allocation units. }\end{array}$ & $\begin{array}{l}\text { Used joint pilot assignment and } \\
\text { resource allocation. } \\
\text { - Used an iterative algorithm to solve } \\
\text { the transformed problem. }\end{array}$ & $\begin{array}{ll}\text { - } & \text { Proposed Algorithm } \\
\text { - } & \text { Conventional scheme. }\end{array}$ \\
\hline $\begin{array}{l}2015 \\
{[12]}\end{array}$ & $\begin{array}{l}\text { To maximize the EE the } \\
\text { receiver. }\end{array}$ & $\begin{array}{l}\text { - Used ADC resolutions of a multi- } \\
\text { antenna receiver }\end{array}$ & $\begin{array}{l}\text { - Impact of quality of } \\
\text { channel estimation should } \\
\text { be carefully investigate. }\end{array}$ \\
\hline $\begin{array}{l}2016 \\
{[13]}\end{array}$ & $\begin{array}{l}\text { Investigated energy } \\
\text { and cost efficiency solutions } \\
\text { for } 5 \mathrm{G} \text { wireless } \\
\text { communication } \\
\text { systems with a large number } \\
\text { of antennas and RF chains }\end{array}$ & $\begin{array}{l}\text { massive MIMO antennas and } \\
\text { millimeter wave technology. } \\
\text { - Investigate EE in multicell } \\
\text { scenarios. }\end{array}$ & $\begin{array}{ll}\text { - } & \text { EEHP Algorithm } \\
\text { proposed. } \\
\text { - } \\
\text { CNAS Algorithm } \\
\text { proposed. }\end{array}$ \\
\hline $\begin{array}{l}2016 \\
{[14]}\end{array}$ & $\begin{array}{l}\text { Evaluate the performance of } \\
\text { user mobility for } 5 \mathrm{G} \text { small cell } \\
\text { networks }\end{array}$ & $\begin{array}{l}\text { - Using individual mobility model } \\
\text { (IMM). }\end{array}$ & $\begin{array}{l}\text { - System model based on } \\
\text { IIM. }\end{array}$ \\
\hline $\begin{array}{l}2016 \\
{[15]}\end{array}$ & $\begin{array}{l}\text { Large cost of RF } \\
\text { circuits }\end{array}$ & $\begin{array}{l}\text { Used energy efficient (EEHP- } \\
\text { MRFC) algorithm } \\
\text { - Used the critical NoA searching } \\
\text { (CNAS) to reduce CPC }\end{array}$ & $\begin{array}{l}\text { - } \quad \text { EE Hybrid Precoding zero } \\
\text { forcing }(\mathrm{ZF}) .\end{array}$ \\
\hline $\begin{array}{l}2016 \\
{[16]}\end{array}$ & $\begin{array}{l}\text { Pilot contamination. } \\
\text { Increased CPC. }\end{array}$ & $\begin{array}{l}\text { Maximized EE under optimised } \\
\text { NoA, number of users, and pilot } \\
\text { sequences. }\end{array}$ & $\begin{array}{ll} & \begin{array}{l}\text { Proposed (AO-BS) } \\
\text { algorithm }\end{array}\end{array}$ \\
\hline $\begin{array}{l}2017 \\
{[17]}\end{array}$ & $\begin{array}{l}\text { To achieve multiple spectral } \\
\text { and EE gains. }\end{array}$ & $\begin{array}{l}\text { - By designing the BSs with several } \\
\text { antennas. }\end{array}$ & $\begin{array}{l}\text { - } \\
\text { RF energy harvesting } \\
\text { capabilities cannot } \\
\text { investigated. }\end{array}$ \\
\hline $\begin{array}{l}2019 \\
{[18]}\end{array}$ & $\begin{array}{l}\text { Inter-cell interference. } \\
\text { High computational } \\
\text { complexity. }\end{array}$ & $\begin{array}{l}\text { Maximized EE under optimal NoA } \\
\text { selection, and user scheduling. }\end{array}$ & $\begin{array}{ll}\text { - } & \text { Adaptive Markov Chain. } \\
\text { Limited policies } \\
\text { generalized to MM } \\
\text { regime. }\end{array}$ \\
\hline
\end{tabular}

1. Network design and implementation: 'Energy efficiency' (EE) is an enormous strategy that immensely declines the circuit power consumption (CPC) in fifth-generation cellular networks. This issue was enhanced because of the increasing number of antennas in massive multiple-input multiple-output (MIMO) systems. The Energy Efficiency of wireless networks increases by the approach of network designing and implementation. It is a technique used to deploy infrastructure nodes to broaden the confined area per consumed energy, comparatively than just the confined area. In addition, the use of base station (BS) switch-on/ switch-off algorithms and antenna muting techniques 


\section{International Journal of Advanced Research in Computer and Communication Engineering}

Vol. 10, Issue 5, May 2021

DOI 10.17148/IJARCCE.2021.105148

to adapt to the traffic conditions, can further reduce energy consumptions. Various technologies have been aimed for the planning implementation and operation of $5 \mathrm{G}$ networks to deal with the immense number of connected devices.

- Extensive Heterogeneous Networks: The concept of extensive networks is a concern with the enormously rising number of devices to serve by expanding the amount of implement infrastructure hardware. The principle fundamentals of network densification can attain strength and emerges as a very strong phenomenon for the implementation of 5G networks. Far from existing network implementation, which consistently decomposes a macrocell into almost less number of smaller areas, each compensates with small base stations, extensive heterogeneous networks significantly enhance the number of infrastructure nodes per unit of area [19]. A huge number of heterogeneous infrastructure nodes differ from macro BSs to femto-cells and relays are opportunistically implemented and stimulated in a demand-based pattern, thus leading to an irregularly shaped network design such as that shown in Fig. 3.

(a)

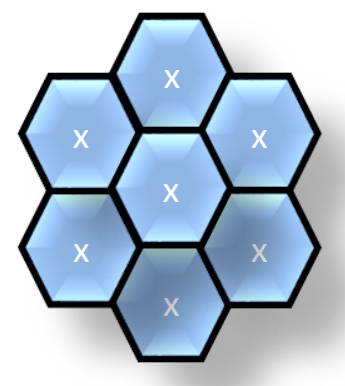

(a.) Conventional layout

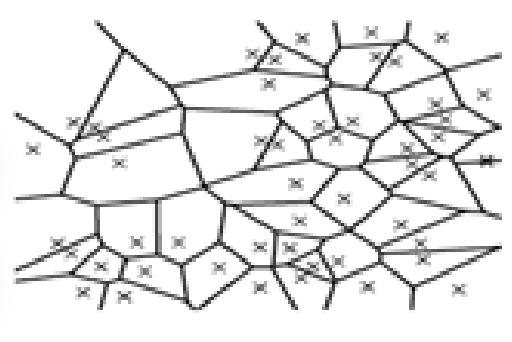

(b)

Fig. 3 Evolution of cellular network layout.

(b.) $5 \mathrm{G}$ layout.

A crucial dispute when dealing with extensive heterogeneous networks is the design of the arrangement of the nodes in the network, which is usually typical to conclude inevitably. Rather, the nodes' locations are designed as random variables with a given spatial distribution, and within this framework, the ultimate extensive used principle is the theory of stochastic geometry [20] use this principle, most study proposed theory on extensive networks has been centered on the evaluation of traditional, non-energy efficient performance measures. Fewer consequences are accessible as much as energy efficiency is involved. From an energy-efficient point of view, node densification diminishes the (electrical and/or physical) separations between communicating terminals, thus managing higher data rates at lower transmit powers. Nevertheless, it still creates additional interference, which might diminish the network energy efficiency. This study has been investigated in [21], where it is clear that densification provides a useful impact on energy efficiency, but the benefit penetrates as the density of the framework nodes increases, thus illustrating that an optimal density level remain exists. The theory proposes the optimal network densification level is [22] examined, where a threshold value on the operating cost of a narrow BS is determined. If the threshold value is less, then micro BSs are profitable; on the other hand, if the threshold value will become more, they can be switched off.

Furthermore in this paper proposes [23], fractional programming to establish a spectrum allocation algorithm in OFDMA heterogeneous networks, to diminish the energy expenditure per transmitted bit. Additionally, another paper [24], investigate exceptional consequences regarding, using a game-theoretic approach, that framework sharing between different mobile network operators (MNOs) may accompany substantial energy savings by boosting the percentage of BSs in sleep mode.

- $\quad$ Massive MIMO: If the concept of dense networks is to modify the number of framework nodes, the belief of massive MIMO is to modify the number of implemented antennas. In massive MIMO, traditional arrays with only minor antennas encouraged by heavy and costly hardware are changed by hundreds of limited antennas, which are maintained by low-cost amplifiers and circuitry. The exploration in such a technology has been stimulated [25], which examined how, incurring the law of giant numbers, large antenna arrays can balance out multi-user interference. This emerges the so-called encouraging propagation condition holds, which has been analytically justified in the overview works [26].

Furthermore, massive MIMO systems also come with certain challenges and destructions. First, expanding a very large number of antenna points in the direction of very enormous systems, for which a microscopic evaluation is consistently too convoluted. Rather, system inquiry and design must complete based on the defining behavior of the network. Thus, this effort can be carried out employing random matrix theory.

Additionally, massive MIMO systems are outline by a more ambitious channel estimation effort, due to a more harsh pilot contamination consequence, and to more additionally serious hardware abolition. Improvements to challenges, 


\section{International Journal of Advanced Research in Computer and Communication Engineering}

Vol. 10, Issue 5, May 2021

DOI 10.17148/IJARCCE.2021.105148

these disputes have mainly directed on conventional performance methods, and outcomes on the energy efficiency of massive MIMO systems have established. As the energy efficiency is involved, massive MIMO has been demonstrated to curtail the radiated power by a component proportional to the square root of the number of deployed antennas, while carrying the information rate unchangeably [27]. Nonetheless, this outcome appeals to an optimal, single-cell massive MIMO system entirely, and without taking into account the hardware-consumed power. However, the accumulated action of hardware damages in massive MIMO systems and the energy efficiency are investigated [28]. Furthermore, the hardware power encompassed in the evaluation and demonstrated that the network energy efficiency is enlarged for a limited number of deployed antennas [29]. Additionally, these exclusive concerns forward the field of energy efficiency in dense networks. Furthermore, this research investigate [30] a structure for self-organizing cells that autonomously activate or deactivate in acknowledgment of traffic demands.

Network formation in feedback to traffic conditions is also investigated [31]. This concluded that in the existence of heavy traffic conditions, deploying indoor small cells is more energy-efficient than conventional network layouts. Additionally, [32] an optimization structure for energy-efficient radio resource management in heterogeneous networks is recognized, considering stochastic traffic advents. Likewise, this research [33] incorporates the heterogeneous path and the massive MIMO technology, examining the complication of regulating the ideal BS density, transmit power levels, and a number of deployed antennas for greatest energy efficiency. Other than this, this research analyst [34] deals with a two-way relay channel, in which multiple combinations of full-duplex users exchange information through a full-duplex amplify and- forward relay with extensive antennas. Contrasting transceiver approaches were analyzed, which are demonstrated to attain energy efficiency gains.

\section{Offloading techniques}

Offloading techniques are a major key technology used to enhance the capacity and energy efficiency of future generation mobile networks. Upcoming networks will tremendously depend upon offloading technique approach. At present, the user devices are earlier equipped with multiple radio access technologies (RATs) such that wherever some other associated technologies are present such that cellular traffic can be offloaded and new cellular resources can be brought to those users that will not offload their traffic. However, the following offloading strategies/approaches can be used to overcome the traffic overload such as Device-to-device (D2D) communications.

- Device-to-device (D2D) communications:A radio technology that facilitates devices to transmit data precisely with other devices, without transmitting the data using a network framework refer to Device-To-Device (D2D) communication. It is the fundamental solution for the next generation (5G) networks to negotiate with these concerns. D2D communication in cellular networks supports straightforward communication among two mobile users without the involvement of a Base Station (BS) or infrastructure-based network. It further plays a vital role in forthcoming cellular networks as it ensures ultra-low latency for communication among users. This innovative approach may operate in a licensed or unlicensed spectrum. It is a novel extension of the conventional cellular communication criterion.

In twhe development of D2D communications, massive MIMO, and small cells for 3GPP LTE Advanced networks has been introduced as an approach of employing the enormous growth in mobile data traffic and decline the large infrastructural cost. While user devices are not acknowledged to directly communicate in a traditional network, D2D communications refer rather to the scenario in which certain co-located devices can connect directly with the help of a cellular frequency. D2D techniques have an intellectual influence on the system energy efficiency as direct transmission among neighboring devices may arise at a much lower transmit power than that desired for communication through a BS that can be far away. The current system design for energy efficiency involves the modern direction of proximity-based applications such as proximity file sharing, advertising, and local multicasting. The D2D communications have been recommended for the enhancement of the flexibility and network throughput of local service, and for reinforcing public safety service in the context of 3GPP LTE-Advanced poor network coverage. In D2D communications, nearby cellular network users can instantaneously share data without passing the BS.

Furthermore, the D2D communications can convince proximity gains, hop gain, and reuse gain. In such a way, device EE can be advanced by D2D communications.

Additionally, it can allow users, more space such that they can share data in any of the three enthusiastic ways. The three ways are as follows: (1) By using the orthogonal resource of the traditional cellular users, (2) transmitting data directly reusing the resource of other cellular users (reusing mode), and (3) is considered as traditional cellular users who can communicate with each other in a typical way through the BS (Cellular mode). Network and device EE can be substantially improved through proper mode selection. Traditional MIMO can accomplish spatial diversity, spatial multiplexing gains, and array gain, and can boost EE. Large-scale or massive MIMO is recognized as a cost-effective infrastructure for $5 \mathrm{G}$ networks because of using more antennas at its BS correlated to the minimal MIMO. The BS in a massive MIMO system in Figure 4, can communicate concurrently with various users on a similar frequency rating. 


\section{International Journal of Advanced Research in Computer and Communication Engineering}

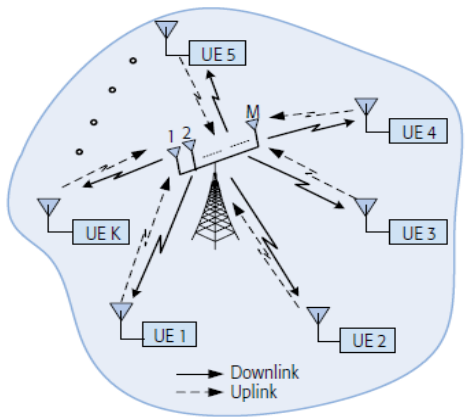

Fig. 4 Massive MIMO communication

When there are several antennas at the BS correlated to the number of users, the channel vectors become asymptotically orthogonal for distinct users. Thus, simple linear precoders such as zero-forcing as well as matched-filter can be deployed to asymptotically bring about the appropriate capacity. To accomplish a corresponding data rate like the single-antenna systems with known perfect CSIT at the BS, the transmit power is reduced by the number set up antennas at the BS. The EE of a system can be increased by disabling some antennas at the base station as in the minimal MIMO. On the other hand, the EE can be enhanced by administering a hybrid analog and digital beamforming RF structure for circuit power balancing and power transmission. To maintain the integrity of the specifications, certain antennas are associated with one RF chain to form analog beamforming that provides the array gain, while certain analog beams are associated to form the digital beamforming that results in the multiplexing gain.

In the heterogeneous network of massive MIMO, improving EE is a big challenge. An area can be under the coverage of a massive MIMO, and from the EE aspect, researchers are still challenging the massive MIMO and HetNets deployment for similar coverage. More flexible and greater EE can be brought by sleep mode control for various small cells in HetNets on the other hand the massive MIMO cell can execute less number of small cells because of its large array gain. Consequently, to study energy-efficient massive MIMO systems in the Heterogeneous networks are required.

\section{IV.CONCLUSION}

In this paper, we have discussed the outline of fifth-generation wireless technology followed by five other major technologies of 5G namely millimeter wave, small cells, Massive MIMO, Beamforming, and Full-duplex respectively. Also, Energy efficiency has now become a fundamental aspect in the framework of communication networks. With the advent of the fifth generation of wireless networks, with millions of increased base stations and billions of connected devices, the insistence for energy-efficient system architecture and operation will be indeed more compelling. Significantly, this paper summarizes the diverse research work that gives an account of energy efficiency comprising the papers published in this special concern. Further, investigating energy-efficient massive MIMO systems in the Heterogeneous networks a leading challenge in the research field.

\section{REFERENCES}

[1]. W. Zhaocheng, C. Qian, L. Dai, J. Chen, C. Sun et al., "Location-based channel estimation and pilot assignment for massive MIMO systems," in IEEE Int. Conf. on Communication Workshop, London, UK, pp. 1264-1268, 2015.

[2]. X. Wenjun, S. Li, S. Wang, Z. Feng, J. Lin et al., "Joint parameter selection for massive MIMO: An energy-efficient perspective," IEEE Access, vol. 4, pp. 3719-3731, 2016.

[3]. W. Liu, S. Han and C. Yang, "Energy efficiency scaling law of massive MIMO systems," IEEE Transactions on Communications, vol. 65, no. 1, pp. 107-121, 2016

[4]. A. Salh, L. Audah, N. S. Shah and S. A. Hamzah, "Mitigating pilot contamination for channel estimation in multi-cell massive MIMO systems," Wireless Personal Communication, vol. 112, no. 3, pp. 1643-1658, 2020.

[5]. A. Salh, N. S. Shah, L. Audah, Q. Abdullah, W. A. Jabbar et al., "Energy efficient power allocation and joint user association in multiuserdownlink massive MIMO system," IEEE Access, vol. 8, pp. 1314-1326, 2020.

[6]. H. Pervaiz, L. Musavian and Q. Ni, "Joint user association and energy-efficient resource allocation with minimum-rate constraints in two-tier HetNets," in IEEE 24th Annual International Symposium on Personal, Indoor, andMobile Radio Communications, London, UK, pp. 1634-1639, 2013.

[7]. D. Ha, K. Lee and J. Kang, "Energy efficiency analysis with circuit power consumption in massive MIMO systems," in IEEE 24th Annual Int. Sym. on Personal, Indoor, and Mobile Radio Communications, London, UK, pp. 938-942, 2013.

[8]. W. Liu, S. Han and C. Yang, "Is massive MIMO energy efficient?," arXiv preprint arXiv:1505.07187, 2015.

[9]. X. Ge, Y. Sun, H. Gharavi and J. Thompson, "Joint optimization of computation and communication power in multi-user massive MIMO systems,” IEEE Transaction on Wireless Communication, vol. 17, no. 6, pp. 4051-4063, 2018.

[10]. Peng, M., Zhang, K., Jiang, J., Wang, J., \& Wang, W. (2014). Energy-efficient resource assignment and power allocation in heterogeneous cloud radio access networks. IEEE Transactions on Vehicular Technology, 64(11), 5275-5287. 


\section{International Journal of Advanced Research in Computer and Communication Engineering}

Vol. 10, Issue 5, May 2021

\section{DOI 10.17148/IJARCCE.2021.105148}

[11]. W. Zhaocheng, C. Qian, L. Dai, J. Chen, C. Sun et al., "Location-based channel estimation and pilot assignment for massive MIMO systems," in IEEE Int. Conf. on CommunicationWorkshop, London, UK, pp. 1264-1268, 2015.

[12]. Bai, Q., \& Nossek, J. A. (2015). Energy efficiency maximization for 5G multi-antenna receivers. Transactions on Emerging Telecommunications Technologies, 26(1), 3-14.

[13]. Zi, R., Ge, X., Thompson, J., Wang, C. X., Wang, H., \& Han, T. (2016). Energy efficiency optimization of 5G radio frequency chain systems. IEEE Journal on Selected Areas in Communications, 34(4), 758-771.

[14]. Ge, X., Ye, J., Yang, Y., \& Li, Q. (2016). User mobility evaluation for 5G small cell networks based on individual mobility model. IEEE Journal on Selected Areas in Communications, 34(3), 528-541.

[15]. R. Zi, X. Ge, J. Thompson, C. X. Wang, H. Wang et al., "Energy efficiency optimization of 5G radio frequency chain systems," IEEE Journal on Selected Areas in Communication, vol. 34, no. 4, pp. 758- 771, 2016.

[16]. H. Q. Ngo, L. N. Tran, T. Q. Duong, M. Matthaiou and E. G. Larsson, "On the total energy efficiency of cell-free massive MIMO," IEEE Transactions on Green Communication and Networking, vol. 2, no. 1, pp. 25-39, 2017.

[17]. Prasad, K. S. V., Hossain, E., \& Bhargava, V. K. (2017). Energy efficiency in massive MIMO-based 5G networks: Opportunities and challenges. IEEE Wireless Communications, 24(3), 86-94.

[18]. J. Hoydis, S. T. Brink and M. Debbah, "Massive MIMO in the UL/DL of cellular networks: How many antennas do we need?," IEEE Journal Selection Areas Communication, vol. 31, no. 2, pp. 160- 171, 2013.

[19]. J. G. Andrews, "Seven ways that HetNets are a cellular paradigm shift," IEEE Communications Magazine, vol. 51, no. 3, pp. 136-144, March 2013.

[20]. F. Baccelli and B. Błaszczyszyn, "Stochastic geometry and wireless networks: Volume I theory," Now Publishers: Foundations and Trends in Networking, vol. 3, no. 3-4, pp. 249-449,

[21]. Y. S. Soh, T. Q. S. Quek, M. Kountouris, and H. Shin, "Energy efficient heterogeneous cellular networks," IEEE Journal on Selected Areas in Communications, vol. 31, no. 5, pp. 840-850, May 2013.

[22]. D. Cao, S. Zhou, and Z. Niu, "Optimal combination of base station densities for energy-efficient two-tier heterogeneous cellular networks,"11 IEEE Transaction on Wireless Communications, vol. 12, no. 9, September 2013.

[23]. S. Kim, B. G. Lee, and D. Park, "Energy-per-bit minimized radio resource allocation in heterogeneous networks," IEEE Transactions on Wireless Communications, vol. 13, no. 4, pp. 1862-1873, April 2014.

[24]. J. Xu, L. Duan, and R. Zhang, "Energy group buying with loading sharing for green cellular networks," IEEE Journal on Selected Areas in Communications, vol. 34, no. 4, April 2016.

[25]. T. L. Marzetta, "Noncooperative cellular wireless with unlimited numbers of base station antennas," IEEE Transactions on Wireless Communications, vol. 9, no. 11, pp. 3590-3600, November 2010.

[26]. F. Rusek, D. Persson, B. K. Lau, E. G. Larsson, T. L. Marzetta, O. Edfors, and F. Tufvesson, "Scaling up MIMO: Opportunities and challenges with very large arrays," IEEE Signal Processing Magazine, vol. 30, no. 1, pp. 40-60, January 2013.

[27]. H. Q. Ngo, E. G. Larsson, and T. L. Marzetta, "Energy and spectral efficiency of very large multiuser MIMO systems," IEEE Transactions on Communications, vol. 61, no. 4, April 2013.

[28]. E. Bj"ornson, J. Hoydis, M. Kountouris, and M. Debbah, "Massive MIMO systems with non-ideal hardware: Energy efficiency, estimation, and capacity limits," IEEE Transaction on Information Theory, vol. 60, no. 11, pp. 7112-7139, November 2014.

[29]. E. Bj”ornson, L. Sanguinetti, J. Hoydis, and M. Debbah, “Optimal design of energy-efficient multi-user MIMO systems: Is massive MIMO the answer?" IEEE Transactions on Wireless Communications, vol. 14, no. 6, pp. 3059-3075, June 2015.

[30]. H. Klessig, D. Ohmann, A. I. Reppas, H. Hatzikirou, M. Abedi, M. Simsek, and G. Fettweis, "From immune cells to self-organizing ultra-dense small cell networks," IEEE Journal on Selected Areas in Communications, vol. 34, no. 4, April 2016.

[31]. M. Fiorani, S. Tombaz, F. Farias, L. Wosinska, and P. Monti, “Joint design of radio and transport for green residential access networks," IEEE Journal on Selected Areas in Communications, vol. 34, no. 4, April 2016.

[32]. B. Zhuang, D. Guo, and M. L. Honig, "Energy-efficient cell activation, user association, and spectrum allocation in heterogeneous networks," IEEE Journal on Selected Areas in Communications, vol. 34, no. 4, April 2016.

[33]. E. Bj”ornson, L. Sanguinetti, and M. Kountouris, "Deploying dense networks for maximal energy efficiency: Small cells meet massive MIMO," IEEE Journal on Selected Areas in Communications, vol. 34, no. 4, April 2016.

[34]. Z. Zhang, Z. Chen, M. Shen, and B. Xia, "Spectral and energy efficiency of multi-pair two-way full-duplex relay systems with massive MIMO," IEEE Journal on Selected Areas in Communications, vol. 34, no. 4, April 2016. 